Laser Chem., 1999, Vol. 19, pp. 161-164

Reprints available directly from the publisher Photocopying permitted by license only
(C) 1999 OPA (Overseas Publishers Association) N.V.

Published by license under

the Harwood Academic Publishers imprint, part of The Gordon and Breach Publishing Group.

Printed in India.

\title{
PUMP PROBE ANISOTROPY STUDIES OF THE PHOTOSYNTHETIC REACTION CENTER
}

\author{
D. C. ARNETT ${ }^{\mathrm{a}, *}$, C. C. $\operatorname{MOSER}^{\mathrm{b}}$, P. L. DUTTON ${ }^{\mathrm{b}}$ \\ and N. F. SCHERER ${ }^{\mathrm{c}, \dagger}$ \\ ${ }^{a}$ Department of Chemistry, \\ ${ }^{\mathrm{b}}$ Department of Biochemistry and Biophysics and Johnson Foundation, \\ University of Pennsylvania, Philadelphia PA 19104; \\ ${ }^{\mathrm{c}}$ Department of Chemistry and the James Franck Institute, University of \\ Chicago, Chicago IL 60637
}

(Received 8 April 1997)

The photosynthetic reaction center of $R b$. sphaeroides is investigated by unique pumpprobe anisotropy methods using $13 \mathrm{fs}$ optical pulses tunable throughout the near IR absorption band. The time and wavelength resolved pump probe anisotropy transients yield detailed experimental descriptions of the energy levels, $P_{y^{+}}$and $P_{y^{-}}$of the excitonically coupled special pair $(P)$, and dynamics prior to charge separation, particularly energy transfer from the accessory bacteriochlorophylla's (B) to the special pair $(P)$.

Keywords: Photosynthesis; energy transfer; anisotropy

Although much work has focused on the dynamics prior to and during charge separation in the photosynthetic reaction center [1], many issues remain unresolved, including the nature of the upper excitonic state of the electronically excited special pair $\left(P_{y^{+}}\right.$, where $P$ is the special pair ground state) and its influence on energy and electron transfer [2, 3]. In this report, we present "two color" wavelength resolved pump-probe anisotropy measurements [4] of the photosyn-

\footnotetext{
*Present address: William R. Wiley Environmental Molecular Sciences Laboratory, Pacific Northwest National Laboratory, Richland WA 99352.

${ }^{\dagger}$ Corresponding author: Tel: 773-702-7069, e-mail: nscherer@rainbow.uchicago.edu
} 
thetic reaction center $(\mathrm{RC})$ of Rhodobacter Sphaeroides at room temperature. The major $Q_{y}$ absorption bands are collectively excited using short $(13-18 \mathrm{fs})$ pulses with a broad spectral range, or individually excited with longer duration, spectrally narrowed pulses that overlap with the transition(s) of interest. A broad-band probe pulse is used to record the response of the system with respect to wavelength, time, and polarization.

Four distinct contributions to the wavelength resolved anisotropy measurements are observed: (i) ground state bleach and (ii) stimulated emission components from $\mathrm{B}$ and $P$, (iii) excited state absorption from $P$, and (iv) a newly identified narrow bleach/stimulated emission band

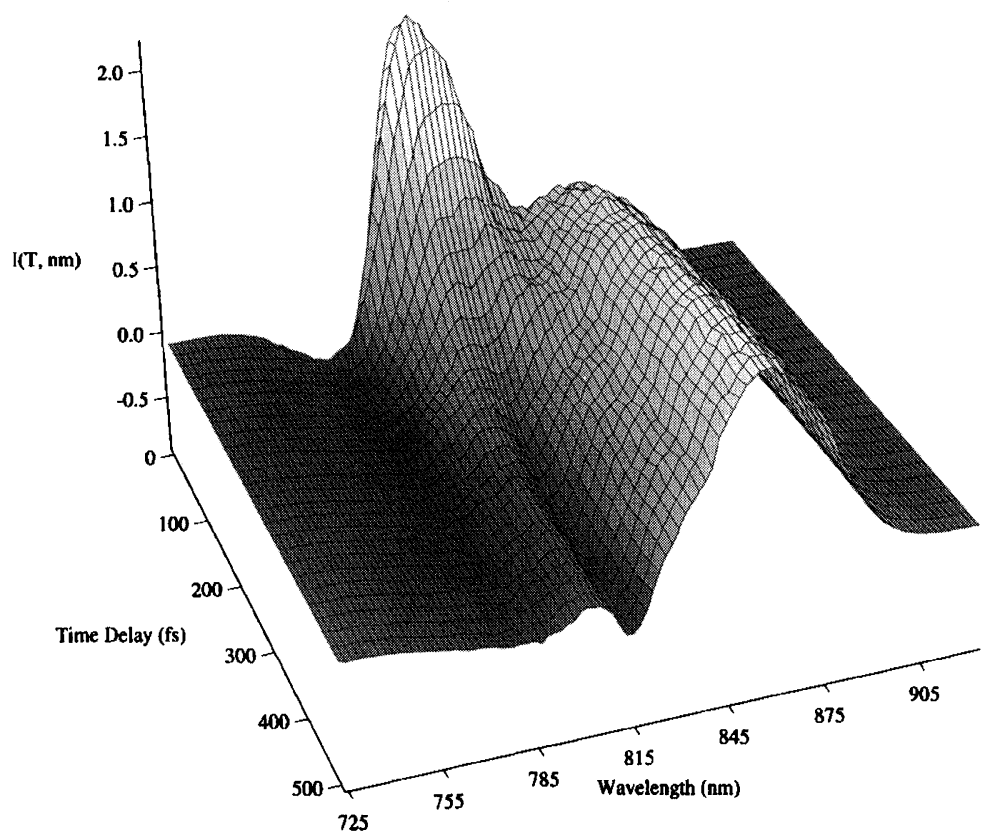

FIGURE 1 The entire temporal and spectral response of the photosynthetic reaction center of $\mathrm{Rb}$. Sphaeroides following excitation of the accessory bacteriochlorophyll pigments (B). Here, a positive signal indicates a transient bleach, while a negative signal indicates a transient absorption. The surface plot shows the rapid decay of a bleach at $800 \mathrm{~nm}$ due to B, and a corresponding increasing bleach at $860 \mathrm{~nm}$ from $P_{y}$. A smaller component at about $825 \mathrm{~nm}$, between these two dominant features, can also be observed and is a associated with $P_{y^{+}}$. The pump-probe relative polarization is at the magic angle. 
at $825 \mathrm{~nm}$, identified as $P_{y^{+}}$. This latter spectroscopic feature appears immediately upon exclusive (i.e., spectrally narrowed) excitation of $P_{y^{-}}$and yields anisotropy values consistent with a transition dipole moment rotated $70^{\circ}$ from the $P-P_{y^{-}}$transition moment, in agreement with low temperature results for $P_{y^{+}}[5,6]$. The absorption maximum of this band, however, occurs at longer wavelengths $(825 \mathrm{~nm}$ $v s .810 \mathrm{~nm}$ at low temperature).

The wavelength resolved magic angle transient observed after excitation of $\mathbf{B}$ is shown in Figure 1. This three-dimensional plot clearly shows the rapid decay of the optically excited state of $\mathbf{B}\left(\mathbf{B}^{*}\right)$ and the subsequent rise of $P_{y^{-}}$following an energy transfer event. The three-dimensional data are analyzed using experimentally determined

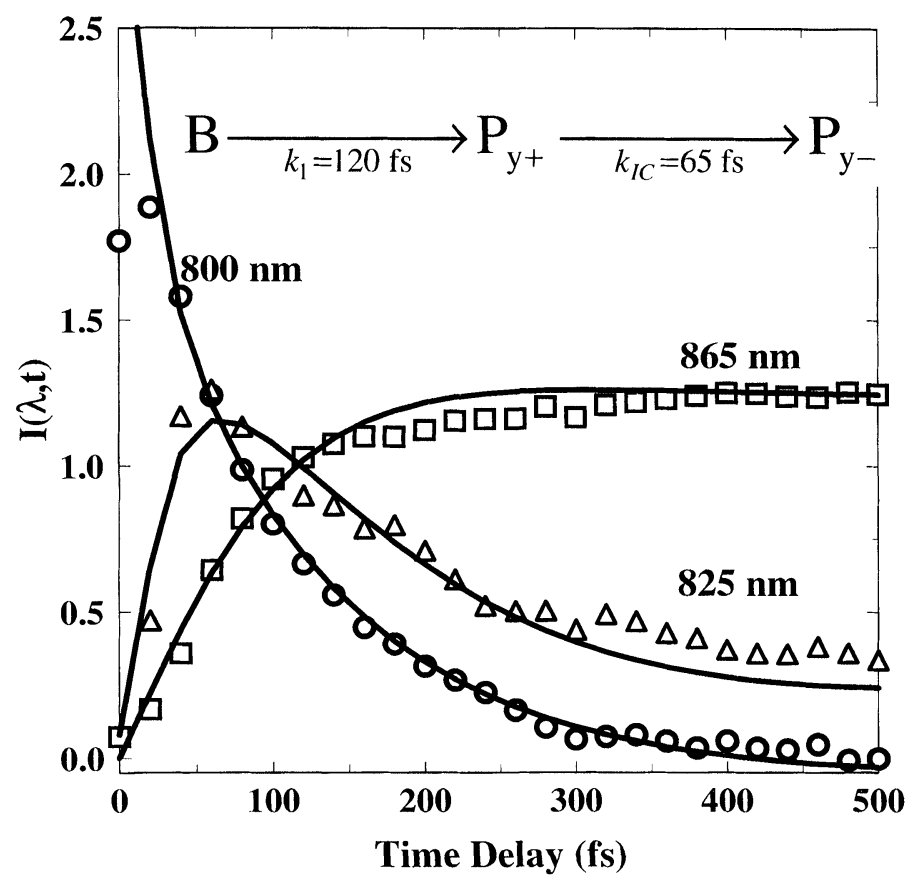

FIGURE 2 Kinetic analysis of the wavelength resolved magic angle pump probe spectra obtained after excitation at $800 \mathrm{~nm}$. The model consists of a two-step energy transfer mechanism, B $\rightarrow P_{y^{+}} \rightarrow P_{y^{-}}$, where the two steps occur with $k_{1}=120 \mathrm{fs}$ and $k_{\mathrm{IC}}=65 \mathrm{fs}$ time constants, respectively, (where IC refers to internal conversion of $P$ ) and the spectra for each species are experimentally determined. Experimental data (symbols) and the calculated fits (solid lines) are shown for 800,825 and $865 \mathrm{~nm}$. 
spectra and anisotropy values for $\mathrm{B}, P_{y^{+}}$, and $P_{y^{-}}$. The detailed structure of the data is satisfactorily fit with a model wherein a very rapid red shift of the initially prepared excited state is followed by energy transfer from B to $P_{y^{-}}$with $P_{y^{+}}$serving as the intermediate. The results of this kinetic analysis are shown in Figure 2. The resulting time constants for the two steps of energy transfer are $120 \mathrm{fs}$ for $\mathrm{B}^{*} \rightarrow P_{y^{+}}$and $65 \mathrm{fs}$ for internal conversion of $P\left(P_{y^{+}} \rightarrow P_{y^{-}}\right)$, the latter of which is independently measured by excitation of $P_{y^{+}}$. These time constants are substantially different than those reported by Jonas et al. [2]. The fast time constants for energy transfer imply substantial electronic overlap between B and $P_{y^{+}}$. Therefore, a supermolecular picture of the reaction center chromophores, i.e., one in which the initial excitation may be delocalized over more than one cofactor, cannot be ignored $[6,7]$.

\section{References}

[1] Fleming, G. R. and Grondelle, R. (1994). Physics Today, pp. 48-55.

[2] Jonas, D. M., Lang, M. J., Nagasawa, Y., Joo, T. and Fleming, G. R. (1996). J. Phys. Chem., 100, 12660-12673.

[3] Stanley, R. J., King, B. and Boxer, S. G. (1996). J. Phys. Chem., 100, 12052-12059.

[4] Wynne, K. and Hochstrasser, R. M. (1993). Chem. Phys., 171, 179-188.

[5] Breton, J. (1985). Biochim. Biophys. Acta, 810, 235-245.

[6] Scherer, P. O. J., Fischer, S. F., Lancaster, C. R. D., Fritzsch, G., Schmidt, S., Arlt, T., Dressler, K. and Zinth, W. (1994). Chem. Phys., 223, 110-115.

[7] Haran, G., Wynne, K. and Hochstrasser, R. (1996). J. Phys. Chem., 100, 5262. 